\title{
WOUND EPITHELIZATION MODEL BY 3D IMAGING
}

\author{
Oindri Ray ${ }^{1}$, Abhisek Adhya ${ }^{2}$, Bijay Kr. Majumdar ${ }^{3}$, Sudhin Ray ${ }^{4}$ \\ ${ }^{1}$ Assistant Professor, Electronics \& Communication Engineering, Meghnad Saha Institute of Technology, West \\ Bengal, India \\ ${ }^{2}$ Researcher .IPGME\&R, Department of Plastic Surgery, S.S.K.M Hospital, West Bengal, India \\ ${ }^{3}$ Professor and head IPGME\&R,Department of Plastic Surgery, S.S.K.M Hospital, West Bengal, India \\ ${ }^{4}$ Research Guide ,IPGME\&R, Department of Plastic Surgery, S.S.K.M Hospital, West Bengal, India
}

\begin{abstract}
Healing by growth of epithelium over a denuded surface is observed by wound assessment process .This study involves the measurement of the amount of wound healing which is done by the simple digital image (taken by SLR digital camera)analysis of particular wound. More than 10 cases are observed with proper skin grafting. Reliability of this assessment process was verified by finding the class of digital image along with interclass correlation. Normally a digital image is a 2-D raster image, which is not containing depth information. This approach is to design a algorithm to detect the detailed process of wound contraction process by modeling a 3D structure. The simple digital image is first determined and grouped by the class of the image following the edge detection and information of pixel to pixel is grouped. Grouping is feasible by choosing the same colour and same spatial placement of the pixel matrix. Pixel to pixel depth dependency is mapped with a matrix using digital image processing toolbox in Matlab. Finally bilateral filtering and rendering will show the $3 D$ contraction model of wound. In conclusion wound epithelization and contraction is a completed process which can be assessed by clinician .This process will help clinicians to detect the amount of healing by producing quantitative measurements. It can be easily compared and studied for different degree and types of wound.Nano silver are used to heal the wound. The wounds are assumed as a denuded surface. The action of nano silver over that surface area is designed as a meshed skin graft integrated with the effect of nano silver crystalline material.
\end{abstract}

Keywords: Wound Assessment, Wound Epithelization, 2-D to 3-D modeling, Digital Image Analysis, Nano silver

\section{INTRODUCTION}

Wound epithelization can be clinically assessed through a simple digital image. This work may be a clinical observation tool by generating a 3D image of burn wound along with its depth level epithelization. Depth mapping algorithm will generate the stereoscopic image similar with the biological vision system. But depth level uniformity would be the major constraint of $2 \mathrm{D}$ to $3 \mathrm{~d}$ conversion approach. Generating a depth proximity relating equation with variety of pixel to pixel adjustment depth value, this constrained can be solved. Due to the false depth and ill position of pixel depth proximity may introduce some positional vectors ${ }^{[7,3]}$. This work is based on blocking of groups and grouping of pixels depending on class. The base image can be treated as a pixel matrix from which a special matrix is formed. Then the depth values are assigned as per the assumed (hypothetically) depth level proximity .After this interclass correlation combined domain and ranged based filtering is applied for depth base image rendering.

\section{METHODS}

This proposed approach can be designed into few steps. A simple digital image of wound is taken as a test sample. The assessment of this clinical trial is performed over the burn wound patient at S.S.K.M hospital, Kolkata, India .It was approved by institutional ethics committee. A prepared nano silver crystalline material is applied over the burn area .The trial had been taken place into different groups, a)deep dermal 3 rd degree burn b) $2^{\text {nd }}$ order burn c) normal burn
.Taking the digital photographs of those burn area the image can be analyzed before and after treatment which shows the betterment. The result can be verified through a histopathological correlation report to check whether any types of infection were documented or not.

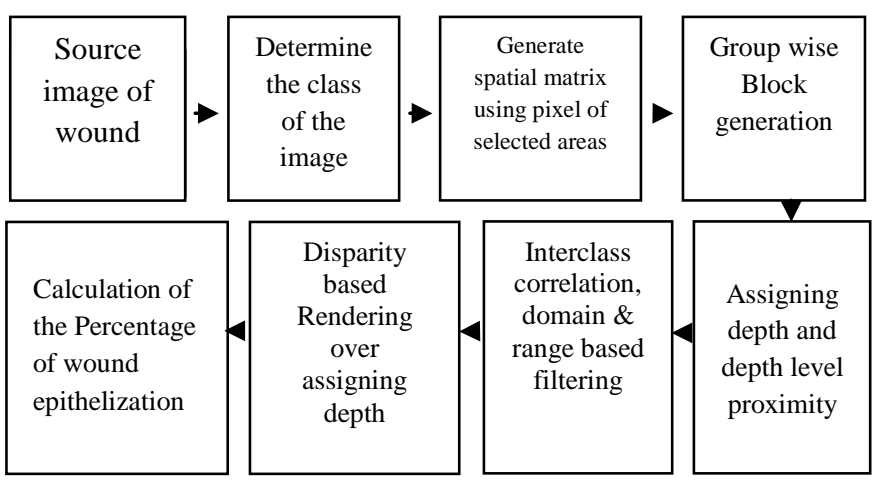

Fig.1.Proposed wound assessment model using 2D image to 3D conversion

\section{MEASUREMENTS}

Determination of class of the image is performed using MATLAB image processing tool box .Spatial matrix of pixel is generated from that wound image and Contracted area and total wound area is marked. The difference in between the contracted area and total wound area is done by the edge information of the image. The same colour pixels are grouped and same spatial matrix value pixels are grouped. More than one similar group forms a block. The 
blocks are connected by the calculation of absolute mean difference of the mean of neighboring blocks. $\mathrm{X}=$ open wound area containing $\mathrm{n}$ number of blocks $\mathrm{Y}=$ Total wound area m number of blocks.

$$
\operatorname{Diff}(\mathrm{X}-\mathrm{Y})=[\operatorname{Mean}(\mathrm{X})-\operatorname{Mean}(\mathrm{Y})]
$$

Mean value represents the mean colour of $\mathrm{X}$ and $\mathrm{Y}$ respectively. A spanning tree is developed to keep the strong edges. Spanning tree algorithm will generate a pathway to make connectivity of same edge pixel in between the spatial matrix formed by same group pixel..Control point selection tool is used to render multi frame image.

\section{GROUP WISE BLOCK GENERATION \&}

\section{GROUP BASED DEPTH LEVEL PROXIMITY}

\section{MEASUREMENT}

From a source digital image of wound two sections should be determined ${ }^{[2]}$. That's why number of pixel of a selected area is measured. The denuded surface area is marked through the edge detection ${ }^{[1,5]}$. Depth Level proximity can be calculated through Depth of pixel= $\mathrm{D}, \mathrm{W}_{\mathrm{d}}=$ width of pixel, $\mathrm{H}_{\mathrm{t}}=$ Height of pixel, $\mathrm{P}_{\mathrm{n}}=$ Pixel Number

$$
\mathrm{D}=\left\{\sum\left[\left(\mathrm{X}-\mathrm{W}_{\mathrm{d}}\right) / 2\right] / \mathrm{W}_{\mathrm{d}}+\left[\left(\mathrm{Y}-\mathrm{H}_{\mathrm{t}}\right) / 2\right] / \mathrm{H}_{\mathrm{t}}\right\} / \mathrm{P}_{\mathrm{n}}
$$

Necrotic or granulation tissues are interpreted as non formed or non developed area or growth is nil over that denuded surface. First section is marked open wound area and total wound area. Marked open wound area is categorized by group blocking $\left(\mathrm{D}_{\mathrm{X}}\right)$ with same colour and same spatial location $^{[3]}$.Total wound area is categorized by group blocking $\left(D_{Y}\right)$. The growth factor of denuded surface is calculated from the formula with interclass co relation

$$
1-\left(\left(D_{X} / D_{Y}\right) X 100 \%\right.
$$

$\mathrm{D}_{\mathrm{X}}=$ Depth level proximity of marked wound area

$\mathrm{D}_{\mathrm{Y}}=$ Depth level proximity of total wound area
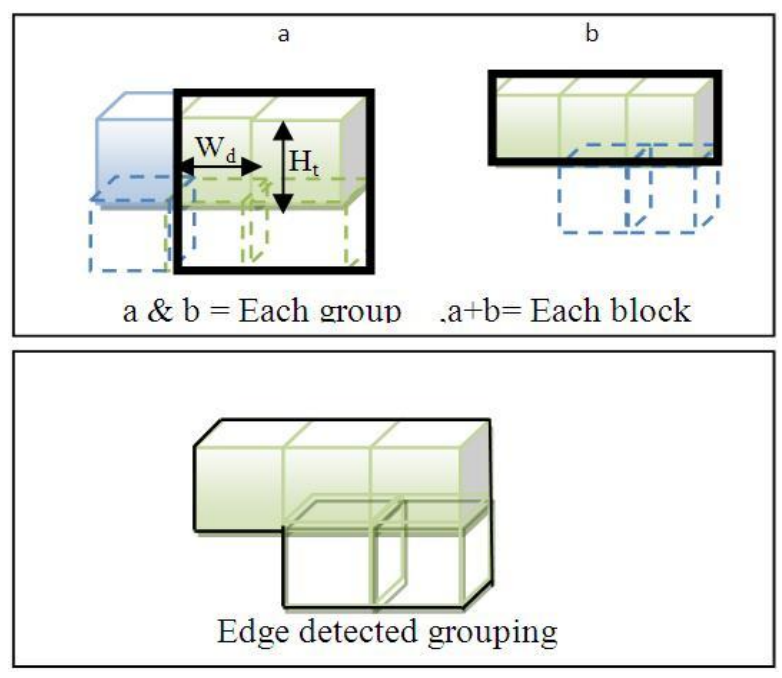

Fig-2: Concept of pixel wise group and block

\section{INTERCLASS CORRELATION, DOMAIN \& RANGE BASED FILTERING}

Combined domain and ranged based filtering is same as the bilateral filtering. After finding the assigned depth level proximity and relating the interclass correlation the depth mapping is feasible ${ }^{[8]}$. After implementation of the pixel spatial matrix the group and block representation is achieved. This filtering replaces the pixel value with an average similar and nearby pixel value ${ }^{[6]}$. It finds the photometric similarity between pixels. For marked area and open area wound filtering is done separately by the following equations

$$
\begin{aligned}
& \mathrm{N}\left(\mathrm{D}_{\mathrm{X}}\right)=\sum \mathrm{e}^{-0.5}\left[\sigma_{\mathrm{X}}{ }^{2}\left\{\mathrm{D}_{\mathrm{X}}(\mathrm{j})-\mathrm{D}_{\mathrm{X}}(\mathrm{i})\right\}\right] \\
& \mathrm{N}\left(\mathrm{D}_{\mathrm{Y}}\right)=\sum \mathrm{e}^{-0.5}\left[\sigma_{\mathrm{Y}}{ }^{2}\left\{\mathrm{D}_{\mathrm{Y}}(\mathrm{j})-\mathrm{D}_{\mathrm{Y}}(\mathrm{i})\right\}\right]
\end{aligned}
$$

Where $N\left(D_{X}\right)$ and $N\left(D_{Y}\right)$ are the normalized factor. $D_{X}(j)$ is the pixel(Matrix) value of open wound area group block and $D_{X}(i)$ is the pixel value of adjacent of $D_{X}(j)$. $D_{Y}(j)$ is the pixel (Matrix) value of total wound area group block. $\mathrm{D}_{\mathrm{Y}}(\mathrm{i})$ is the adjacent pixel value of $D_{Y}(j) . \sigma_{X}, \sigma_{Y}$ are the pixel intensity of open wound area group block and total wound area group block respectively.

\section{DISPARITY BASED WOUND IMAGE RENDERING TO ESTABLISH THE EPITHELIZATION}

This system produces ${ }^{[5,6]}$ the $3 \mathrm{D}$ position of each single observation point $\mathrm{P}$ from its projections, $\mathrm{P}_{\mathrm{L}}$ and $\mathrm{P}_{\mathrm{R}}$.

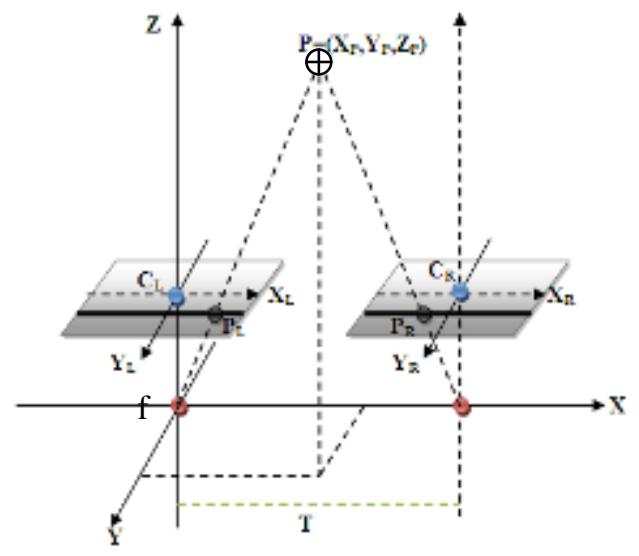

Fig.3.Disparity based image analysis

$$
\mathrm{Z}=\mathrm{fT}
$$

Where $\mathrm{T}=$ distance along the camera $\mathrm{Z}$ axis, $\mathrm{f}=$ focal length (in pixels), $\mathrm{T}=$ baseline(in metres), $\mathrm{d}=$ disparity(in pixels $)=\left(\mathrm{X}_{\mathrm{L}}-\mathrm{X}_{\mathrm{R}}\right)$. After $\mathrm{Z}$ is determined, $\mathrm{X}$ and $\mathrm{Y}$ can be calculated using the usual projective camera equations:

$$
\begin{aligned}
& X=u Z / f \\
& Y=v Z / f
\end{aligned}
$$


Where $u$ and $v$ are the pixel location in the 2D image's, Y, Z is the real $3 \mathrm{D}$ position.

$$
\begin{aligned}
& Z_{P}=f\left(T / X_{L}-X_{R}\right) ; \\
& X_{P}=X_{L}(T / d) ; \\
& Y_{P}=Y_{L}(T / d)
\end{aligned}
$$

\section{RESULT OBTAINED}

The Result of interclass correlation has been achieved by Interclass Co relation equation (3)

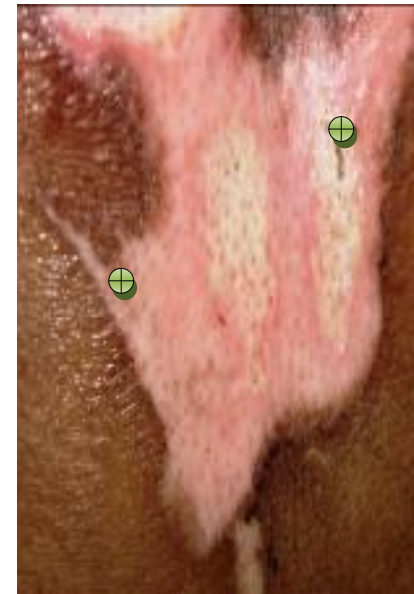

(a)

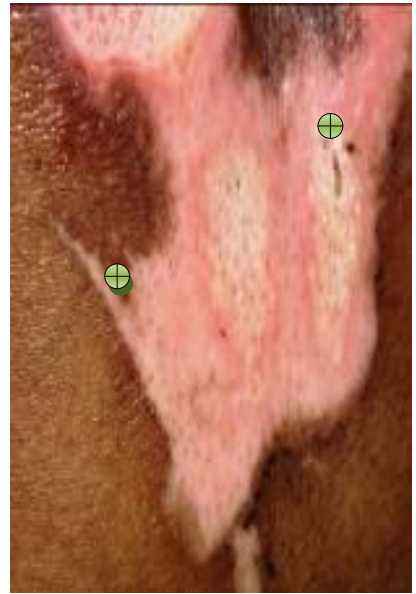

(b)
Fig.4 Disparity Based Image analysis (a) left frame (b) Right frame. $\oplus$ :Matched Point of multi frame Case-1

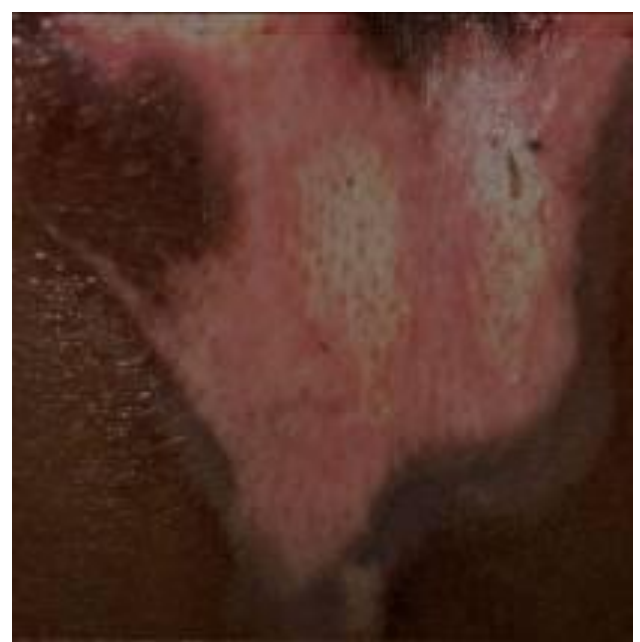

(c)

Fig.5. Assigned depth based rendering over left Frame and right Frame - 3D visualization on Fig.5(a) and (b)

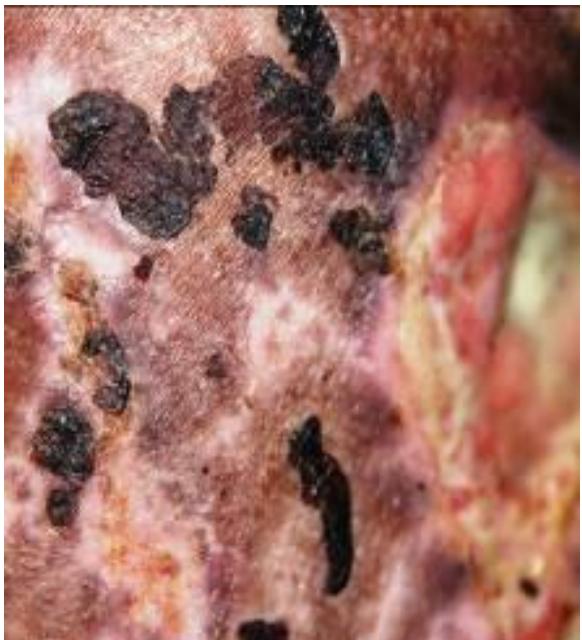

(d)

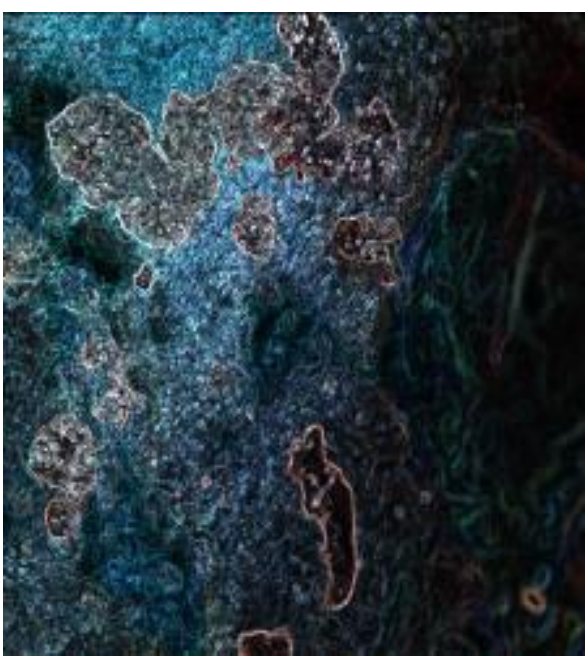

(e)

Fig.6.The Total wound area, open wound pixel information has been detected and edge information is used for determination of Epithelization level.(d) original image (e) After edge detection .Case-2

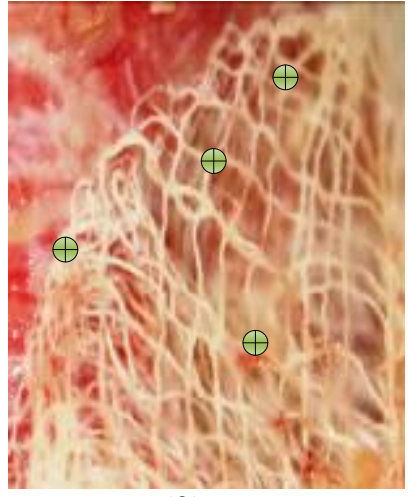

(f)

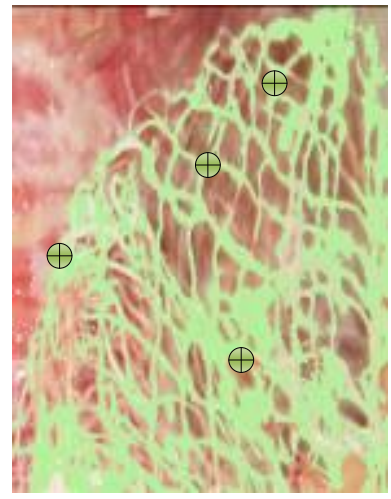

$(\mathrm{g})$
Fig.7. Burn wound (f) area along with overlying split skin graft ${ }^{[11]}$. Open wound area (green) and total wound area is differently marked, from edge information and disparity matching points-Case-3 
Table -1: Wound Digital Image analysis and clinical assessment

\begin{tabular}{|l|l|l|l|}
\hline $\begin{array}{l}\text { Wound } \\
\text { Type } \\
\text { Example }\end{array}$ & $\begin{array}{l}\text { Total } \\
\text { wound } \\
\text { area in } \\
\left.\text { pixel( } \mathrm{D}_{\mathrm{Y}}\right)\end{array}$ & $\begin{array}{l}\text { Open } \\
\text { Wound } \\
\text { area in } \\
\text { pixel }\left(\mathrm{D}_{\mathrm{X}}\right)\end{array}$ & Epithelization \\
\hline Case 1 & 3740000 & 220567 & $94.1 \%$ \\
\hline Case 2 & 2787000 & 210012 & $92.46 \%$ \\
\hline Case3 & 20198772 & 250891 & $98.7 \%$ \\
\hline ST.DEV & 9789188 & 21221.24 & 0.032349 \\
\hline The P value : The statistical significance 0.04176 \\
\hline
\end{tabular}

\section{STATISTICAL ASSESSMENT}

This calculation gives single and mean measuring value of interclass correlation over epithelization level. The Fisher Test, statistical significant $\mathrm{P}$ value values between 0.04 to 0.07 stands for fair to good agreement ${ }^{[14]}$.The standard error is calculated from chi test by showing expected range of epithelization and achieved level of epithelization .

\section{CONCLUSIONS}

Images can be thought of in three dimensions, the $\mathrm{x}$ - and $\mathrm{y}$ axes represent pixel positions and the $\mathrm{z}$-axis represents the intensity of each pixel. By assuming this the intensity values represents the angle of elevations, like topography. The areas of high intensity and low intensity in an image, peaks and valleys in topographical terms, can be important morphological features. This three different cases were observed .The reliability of epithelization with the digital image is statistically established .The percentage of epithelization and wound contraction is measured and categorized by the mean value of three different cases .The skin graft is been performed by minute marking and care. The total research is based on the how a simple digital image will help to categorize a dermal burn wound. The proposed approach will create a digital $3 \mathrm{~d}$ morphology of wound surface .The function of Nano silver can be observed depending upon the epithelization level .This process could be the detailed tool and inter observer reliable system.

\section{REFERENCES}

[1]. Krasner D.Wound Healing Scale,version 1.0:a proposal.Adv Wound Care 1997;10(5):82-5.

[2]. Thomas DR, Rodeheaver GT,Bartolucci AA,Franz RA,Sussman C,Ferrell BA,Etal.pressure ulcer scale for healing :derivation and validation of the PUSh tool.The PUSH task Force.Adv.Wound care 1997;10(5):96-101.

[3]. Hauser J,lehnhardt M,daigeler A,Langer S,Steinau HU,Vogt PM.Photoplanimetric evaluation and impedance measurement of split-thickness skin grafts:a new model for objective wound-healing assessment in clinical trials.Skin Res Technol 2009;15(2):168-71

[4]. Kundin JI.A new way to size up a wound.Am J Nurs 1989;89(2):206-7.

[5]. Lucas C,Classen J,Harrison D,De H.Pressure ulcer surface area measurement using instant full scale photography and transparency tracings. Adv skin wound care 2002;15(1):17-23.

[6]. Y-L chang,et al,'Depth Map Generation for 2D to 3D conversion By Short Term Motion Assisted Colour Segmentation "in processing of ICME,2007

[7]. W.J Tam and L Zhang :3D TV content generation : 2D to 3D conversion,"in Proc. ICME,pp.1869-1872,2006

[8]. Hayward PG, Hillman GR, Quast MJ, Robson MC. Surface area measurement of pressure sores using wound moldsand computerized imaging. J Am Geriatr Soc 1993;41(3):238-40.

[9]. Danielsen P, Jorgensen B, Karlsmark T, Jorgensen LN, AgrenMS. Effect of topical autologous platelet-rich fibrin versusno intervention on epithelialization of donor sites and meshed split-thickness skin autografts: a randomized clinical trial. Plast Reconstr Surg 2008;122(5):1431-40.

[10]. Smith RB, Rogers B, Tolstykh GP, Walsh NE, Davis Jr MG, Bunegin L, et al. Three-dimensional laser imaging systemfor measuring wound geometry. Lasers Surg Med 1998;23(2):87-93.

[11]. Stone CA, Wright H, Clarke T, Powell R, Devaraj VS. Healingat skin graft donor sites dressed with chitosan. Br J Plast .Surg 2000;53(7):601-6.

[12]. Scheres, S.H., 2012. RELION: implementation of a Bayesian approach to cryo-EM,structure determination. J. Struct. Biol. 180, 519-530.

[13]. Zhang, X., Zhou, Z.H., 2011. Limiting factors in atomic resolution cryo electron microscopy: no simple tricks. J. Struct. Biol. 175, 253-263.

[14].Fleiss JL.Statistical method for rates and proportion. New York :wiley; 1981

\section{BIOGRAPHIES}

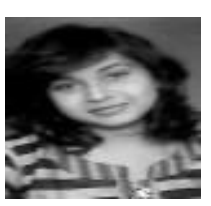

Assistant professor at Meghnad saha Institute of Technology. Researcher, Calcutta University .West Bengal, India

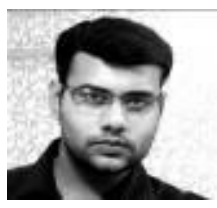

Researcher, IPGME\&R, S.S.K.M Hospital. Kolkata. West Bengal, India

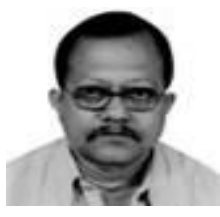

Professor, Head, plastic surgery, S.S.K.M hospital, West Bengal, medical education service

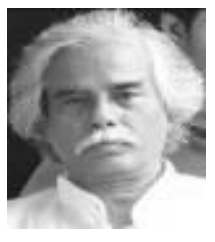

India 